\title{
EXCISION OF RETROCALCANEAL SPUR BY A LATERAL APPROACH FOR RELIEF OF HEEL PAIN.
}

Nandivada V .S . Kiran Kumar

1. Assistant Professor. Department of Orthopaedics, King George Hospital. Visakhapatnam.

CORRESPONDING AUTHOR:

Nandivada V .S . Kiran Kumar,

Assistant Professor,

Department of Orthopaedics,

King George Hospital. Visakhapatnam.

E-mail: drkiranindia@gmail.com

ABSTRACT: retrocalcaneal pain could be due to various reasons depending on the structure involved in the region. Bursal irritation is a common finding which is well relieved by non surgical modality of treatment rest, ice therapy, and other modalities of physiotherapy. CONTEXT (background): Podiatric or foot problems are increasing in our lifestyles due to various reasons . HEEL PAIN DUE TO RETROCALCANEAL SPUR IS AN IMPORTANT CAUSE AMONGST THE MANY CAUSES OF HEEL PAIN Retrocalcaneal bursitis is a common presentation which is easily identifiable and treated. The retrocalcaneal spur or bony osteophyte poses a potential problem to the tendon of tendo achillis and results in its repeated irritation causing tendinitis and future weakening. Hence this study of 5 cases showed that the excision of the retrocalcaneum provided greater relief and i feel the lateral approach to the calcaneal spur area more comfortable . AIM: Aim of this case study is to inform the orthopaedic fraternity and the post graduate students about the pain relief obtained by excision of the retrocalcaneal spur by a lateral approach to the ankle postero lateral to the tendo achillis .MATERIAL AND METHODS : Between 2011 and 2013 an analysis of the retrocalcaneal spur patients and their resistant heel pain cases were given a detailed $\mathrm{x}$ ray examination and were found to have an impinging retrocalcaneal spur and the method of treatment used was a lateral approach to the heel and excision of the posterior retrocalcaneal spur under direct vision .

\section{Statistical analysis used:}

pain due to a bursa around the heel : 4 cases .

pain due to a posterior calcaneal osteophyte : 2 cases.

pain due to retrocalcaneal spur : 5 cases.

\section{Clinical symptoms :}

* pain on walking especially inclined floors and

roads .

* posterior swelling bony to feel,

* activity decreased after exercise,

* EQUINUS from gastro soleus spasm .

* gait is antalgic , excessive pronation of the hind foot

with early heel rise in the gait cycle.

\section{Investigations: $x$ rays of the ankle .}


special views are necessary to see the true extent of spurring .

Lateral view medial oblique view, lateral oblique view.

CONSERVATIVE THERAPY PROTOCOL FOR THE PATIENTS FOLLOWED: 3 Weeks of rest, ice water immersion, plantar flexion splint during night to reduce pressure on the tendo achillis were advised and followed by the patients . out of 11 patients 5 were resistant to all modalities of treatment hence advised spur excision to improve the quality of life.

Incisions used in the excision procedure for retrocalcaneal spurs in the records till date are :

1) Fowler \& Phillip transverse incision over the insertion of the tendo achillis .

2) Lateral linear incision.

3) Medial lazy incision.

4) combined medial and linear incision.

5) Linear midline incision.

In this study a lateral linear incision was used with convenience.

RESULTS: postoperative pain decreased gradually in 7 days as gauzed by von baker pain scale score from grade 8 to grade 2 in all 10 patients as observed on the 10th post operative day and after two months they were having no pain at all . . No wound breaks down and no infections were noted.

CONCLUSIONS: Retrocalcaneal spur causes chronic irritation of the tendo achillis and surgical excision from the lateral approach is a convenient way of eradicating the problem without causing minimum morbidity.

\section{KEYWORDS: retrocalcaneal osteophyte, bursitis.}

MESH TERMS: bursitis, retrocalcaneal spurs , heel pain.

INTRODUCTION OF THE CASE STUDY : Purpose of the study is to highlight the difficulties of understanding heel pain which has been down the line, treated by various modalities like local hydrocortisone treatment, physiotherapy, splinting, various surgical procedures.

The Rationale for the study of these cases is to prove that there is definitive role for the excision of the retrocalcaneal spur by a lateral approach with minimum side effects and complete relief from the heel pain if rightly diagnosed.

MATERIAL AND METHODS: 11 heel pain subjects in middle age and the elderly were selected who failed to get relief from conservative modalities of treatment like ice therapy, local hydrocorticosteroid injections, and other modalities. There is no sex preponderance. Of them 5 patients showed a retrocalcaneal spur on their $\mathrm{x}$ rays. 


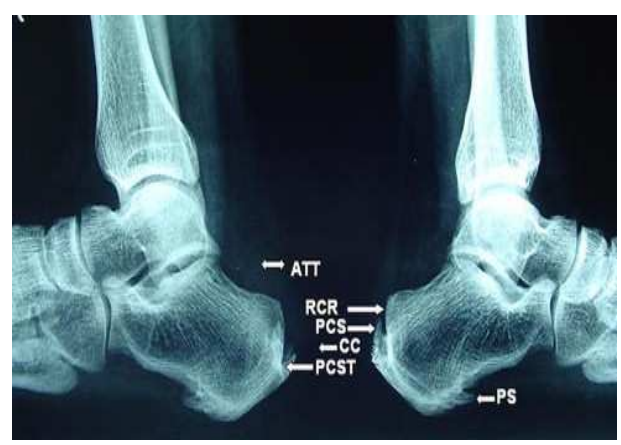

$\mathrm{x}$ ray showing the retrocalcaneal spur growing and causing in pain at the insertion of the tendo achillis in a patient .

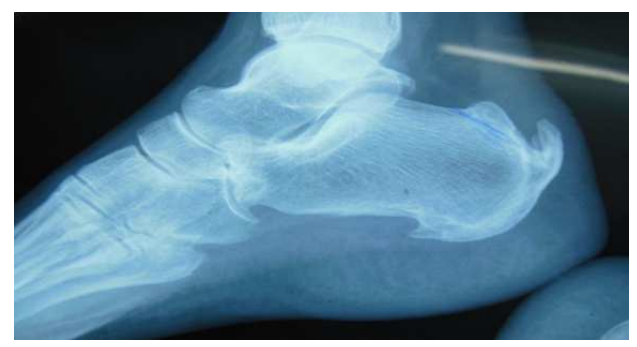

Calcaneal osteophyte plus retrocalcaneal spur seen in this patient in a preoperative case

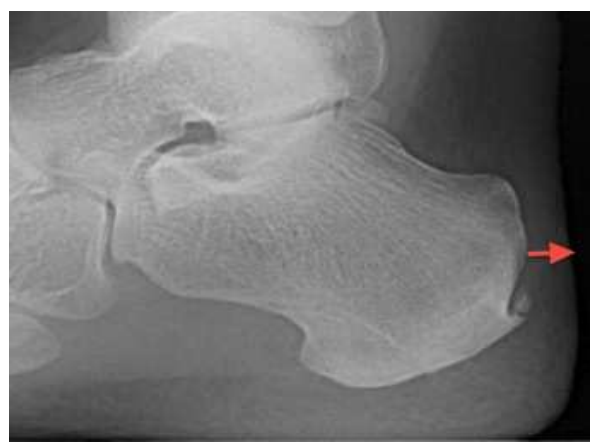

This posteriorly placed protrusion of the retrocalcaneal spur produces friction on the $\mathrm{T} A$ tendon.

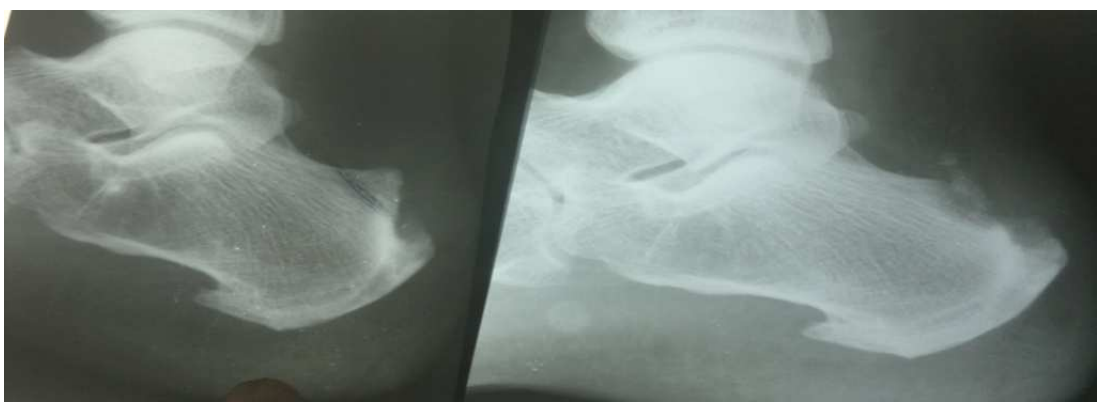

\section{Before after excision}


AETIOLOGY: Young adults with increased activity, and the middle aged and elderly with obesity, wrong fitting shoe wear suffer from this disease .

Clinical symptoms: * pain on walking especially inclined floors and roads .

* Posterior swelling bony to feel,

* Activity decreased after exercise,

* EQUINUS from gastro soleus spasm.

* Gait is antalgic , excessive pronation of the hind foot with early heel rise in the gait cycle.

Local findings : a painful Swelling near the tendo achillis insertion, local warmth and tender bony prominence
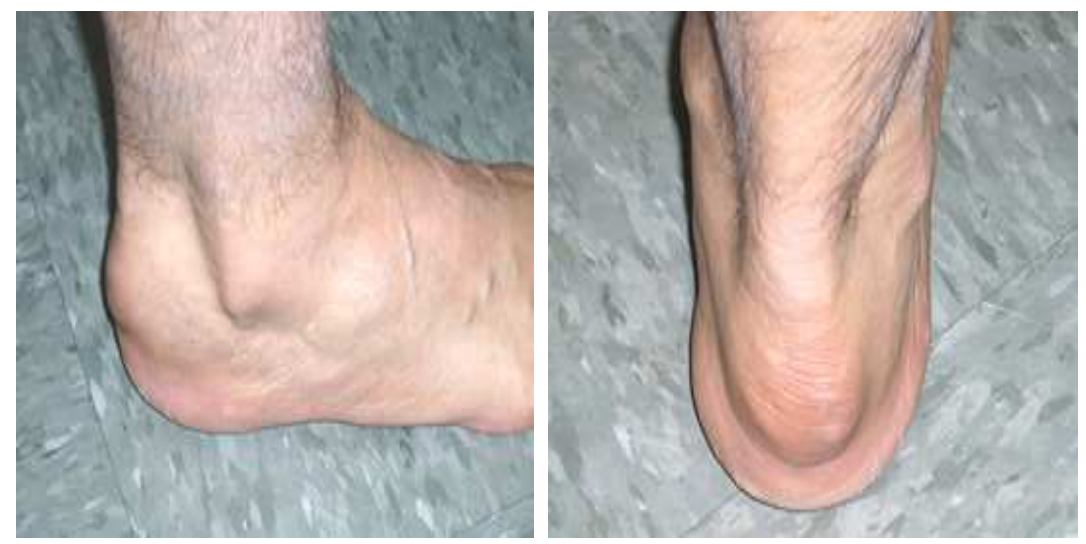

\section{Associated conditions: hypothyroidism in one female patient.}

Options of management: only after THREE weeks of conservative management the patients were taken up for surgery. protocol for conservative therapy included three weeks of Ice therapy , gradual stretching exercises , plantar flexion splints at night to relax the tendo achillis , and then the patient was labeled as a non responder if there was no relief and they were advised and taken up for surgery. Out of 11 patients 5 were resistant to all modalities of treatment hence advised spur excision to improve the quality of life.

Incisions used in the excision procedure for retrocalcaneal spurs in the records till date are :

1) Fowler \& Phillip transverse incision over the insertion of the tendo achillis.

2) Lateral linear incision.

3) Medial lazy incision .

4) combined medial and linear incision.

5) Linear midline incision .

\section{In this study a lateral linear incision was used with convenience.}

RESULTS: postoperative pain decreased gradually in 7 days as gauzed by von baker pain scale score from grade 8 to grade 2 in all 5 patients as observed on the 10th post operative day and after two months they were having no pain at all i.e.

Grade 0 .. No wound break down and no infections were noted. 


\section{UNIVERSAL PAIN ASSESSMENT TOOL}

This pain assessment tool is intended to help patient care providers assess pain according to individual patient needs. Explain and use 0-10 Scale for patient self-assessment. Use the faces or behavioral observations to interpret expressed pain when patient cannot communicate his/her pain intensity.

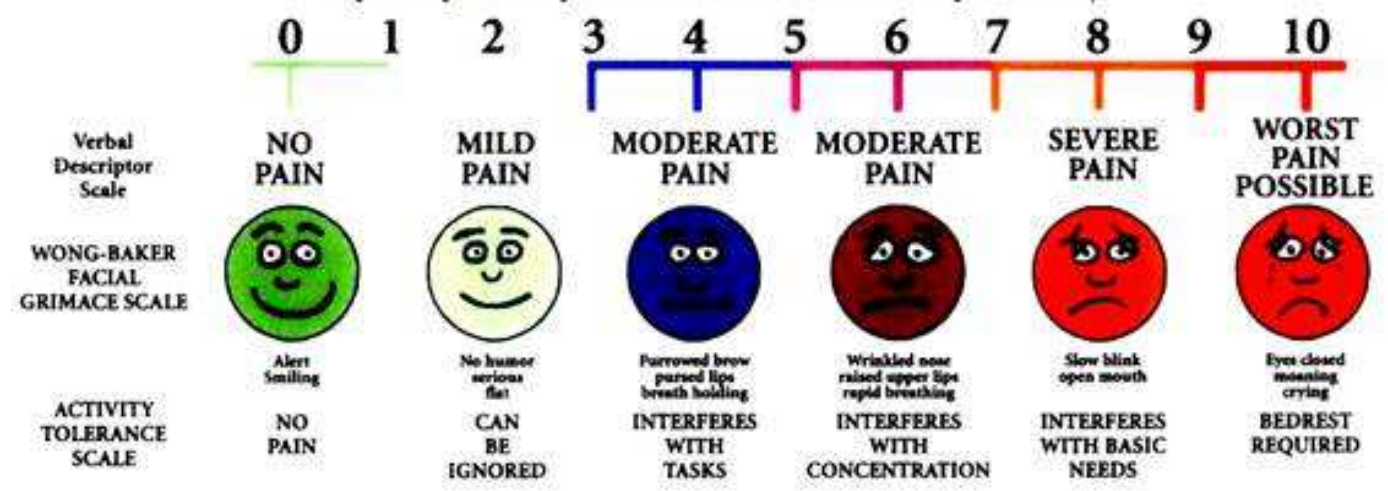

DISCUSSION: In this case study deviation from the direct posterior incision or the medial incision was preferred by using a direct lateral incision as this is the better and direct approach

In between 2012 and 2013 at Krushi orthopaedic welfare society i treated 11 patients with heel pains and 6 of them were found to have bursitis relieved by rest .cold and warm water therapy with postural splints and NSAIDs for a few weeks along with physiotherapy .heel pain can be caused by many causes which are by structures around the calcaneal insertion of the tendo achillis as follows: Achillis tendon tendinitis, retro calcaneal region, posterior calcaneal spur, plantar spur.

But 5 of them were having persistent pain and their x-rays revealed a prominent calcaneal spur which was found to be actually impinging on the tendo achillis tendon irritating it and resulting in its thickening but in reality weakening it due to the chronic friction of every day 's usage of the foot. Excision of the osteophyte is more easier and direct if the lateral approach was used instead of approaching the osteophyte by cutting across the tendo achillis itself and suturing it back as described in many papers across the world as follows:

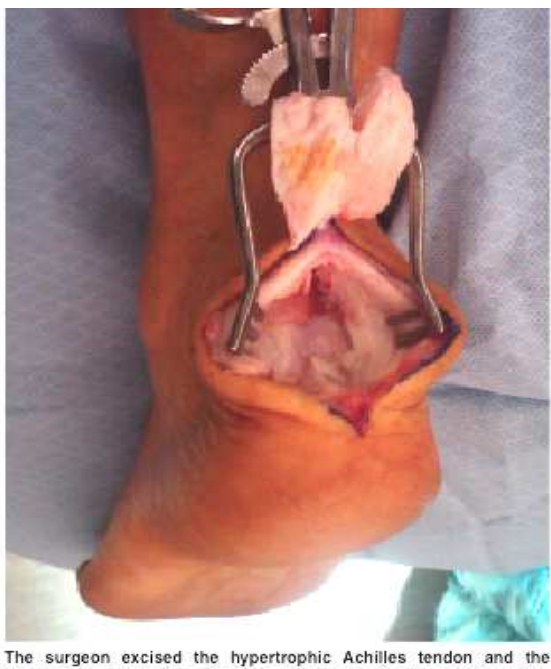

The surgeon excised the hypertrophic Achilles tendon and the
inflamed bursa was visible in the proximal margin of the surgical site.

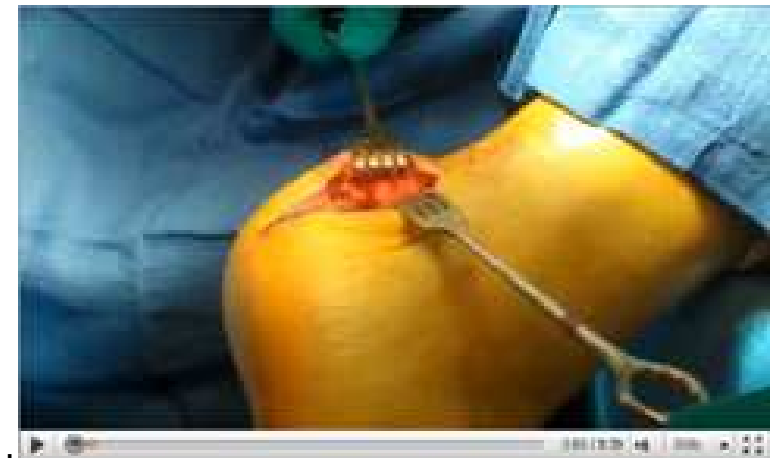

Retrocalcaneal spur could be a cause of a chronic pain in the heel with an old history of painless swelling initially but which became painful with time as it caused tendinitis and bursitis. calcaneal 
osteophyte could form along the insertion of the tendo achillis or a bony spur could develop at the superoposterior corner in front of the tendo achillis tendon .

AUTHORS PROCEDURE: I did a schelp and leach procedure ( cambell text book of operative procedures ) of excising the protrusion and clearing the soft tissue and the bursa around and by direct closure producing complete pain relief to the patient BY A LATERAL LINEAR INCISION WHICH IS EASIER AND CONVENIENT..

CONCLUSION :Retrocalcaneal spur cannot be prevented but once formed they continue to grow and at a certain stage after enough physiotherapy the surgeon has to take a decision whether the pain would be better relieved by a surgical approach and the procedure is surgical excision of the spur and the lateral approach is the best in my ongoing study ,because the tendo achillis is not cut and not threatened in any way and is a simpler learning curve for the juniors too .

REVIEW OF LITERATURE: revealed a paper by denis e martin D.PM where interesting insights into the related problems which need to be understood like the osteotomy of the calcaneum and changing the lever arm of the TA decrease the equinus deformity due to tight gastrosoleus were described where he describes aptly the static and dynamic deforming forces, which can be searched forin http://www.podiatryinstitute.com/pdfs/Update_1995/1995_03.pdf

ACKNOWLEDGMENTS: Acknowledgments to Krushi orthopaedic welfare society for sponsoring this case study Consent of the patients was taken for the publication of the case studies and acknowledgements to all of them .

\section{REFERENCES:}

1. chaow. deland jr bates je et al achillis tendon insertion: an invitro anatomical study foot and ankle journal 18: 81-84;1997.

2. fowler .a.philip jf : abnormalities of the calcaneus as a cause of painful heel: bone and jint surgery 32: 494-500: 1945.

3. 3)gerken ap,mc garvey wc baster de insertional achillis tendinitis :foot and ankle clinics 1:237-249: 1966.

4. 4) pavlow .h. heneghan ma hersa et al the haglund syndrame :initial and differential diagnosis . diagnotic radiology 14: 83- 881982.

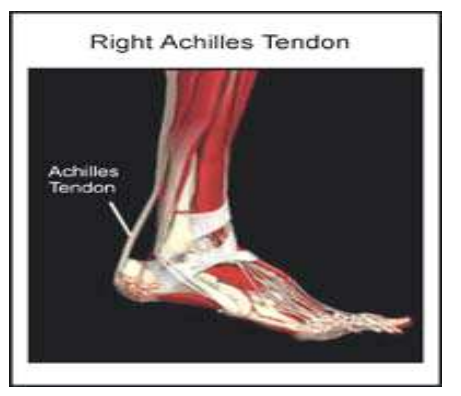

A CASE STUDY OF 5 CASES BETWEEN 2012 AND 2013: 